\title{
Precision electroweak physics at the LHeC and FCC-eh
}

\author{
Daniel Britzger, ${ }^{a}$ Max Klein $^{b}$ and Hubert Spiesberger ${ }^{c}$ \\ ${ }^{a}$ Max-Planck-Institut für Physik, Föhringer Ring 6, 80805 München, Germany \\ ${ }^{b}$ University of Liverpool, Oxford Street, UK-L69 7ZE Liverpool, United Kingdom \\ ${ }^{c}$ Institut für Physik, Johannes-Gutenberg-Universität, Staudinger Weg 7, 55099 Mainz, Germany
}

The proposed electron-proton collider experiments LHeC and FCC-eh at CERN are the highest resolution microscopes that can be realised in the present century and they would represent a really unique research facility. We exploit simulated neutral-current and charged-current deep-inelastic scattering data of the $\mathrm{LHeC}$ and the FCC-eh and examine their sensitivity for precision physics in the Electroweak sector of the Standard Model (SM), like the effective weak mixing angle $\sin ^{2} \theta_{\mathrm{W}, \ell}^{\text {eff }}$, or the light-quark weak-neutralcurrent couplings. Unique measurements are further feasible at high precision for the running of the weak mixing angle, as well as for electroweak effects in charged current interactions. The sensitivity to beyond SM effects is studied using the generic $S, T$ and $U$ parameterization. The report summarizes previous studies about the $\mathrm{LHeC}$ and presents new prospects for the FCC-eh.

Proceedings submitted to the EPS Conference on High Energy Physics (EPS-HEP2021), Hamburg, Germany, 26-30 July 2021 


\section{Introduction}

The LHeC [1, 2] and FCC-eh [3] are proposed electron-proton colliders at CERN with center-of-mass energies of $1.3 \mathrm{TeV}$ or $3.5 \mathrm{TeV}$, respectively. Unlike HERA, or other previous proposals, they are designed as high-luminosity colliders with a targeted integrated luminosity that exceeds $\mathcal{L}>1 \mathrm{ab}^{-1}$, and thus provides great new opportunities for precision measurements in deep-inelastic electron-proton scattering at high energy scales.

In this report, we study simulated inclusive neutral- and charged-current deep-inelastic scattering (NC and CC DIS) data in electron-proton collisions with respect to their sensitivity to parameters of the electroweak interaction in the Standard Model (SM) or possible generic extensions. The process of NC and CC DIS are mediated through spacelike momentum transfer and therefore such measurements are complementary to measurements in the timelike domain in $e^{+} e^{-}$or $p p$ collisions.

After a brief recapitulation of the formalism, we present new prospects for the FCC-eh and compare them with LHeC expectations from Refs. [2, 4].

\section{Electroweak physics in DIS}

At the LHeC and FCC-eh, inclusive NC and CC DIS cross sections will be measured in $e p$ collisions as a function of $Q^{2}$ and $x$, where $Q^{2}$ is the virtuality of the exchanged boson and $x$ is the Bjorken scaling variable. These data will be compared to predictions in the framework of the SM. In the following, we will briefly sketch the calculation of the NC DIS cross sections, and where higher-order Electroweak (EW) or QCD corrections contribute. For details of CC scattering, we refer to Ref. [4].

Inclusive NC DIS cross sections are expressed in terms of generalized structure functions $\tilde{F}_{2}^{ \pm}, x \tilde{F}_{3}^{ \pm}$and $\tilde{F}_{\mathrm{L}}^{ \pm}$at $\mathrm{EW}$ leading order (LO) as

$$
\frac{d^{2} \sigma^{\mathrm{NC}}\left(e^{ \pm} p\right)}{d x d Q^{2}}=\frac{2 \pi \alpha^{2}}{x Q^{4}}\left[Y_{+} \tilde{F}_{2}^{ \pm}\left(x, Q^{2}\right) \mp Y_{-} x \tilde{F}_{3}^{ \pm}\left(x, Q^{2}\right)-y^{2} \tilde{F}_{\mathrm{L}}^{ \pm}\left(x, Q^{2}\right)\right],
$$

using the fine structure constant $\alpha$, the Bjorken scaling variable $x$, the inelasticity $y$, and the factors $Y_{ \pm}=1 \pm(1-y)^{2}$ express the helicity dependence of the process. The generalized structure functions represent the contributions from pure $\gamma$ - and $Z$-exchange, and its interference [5] like

$$
\begin{aligned}
& \tilde{F}_{2}^{ \pm}=F_{2}-\left(g_{V}^{e} \pm P_{e} g_{A}^{e}\right) \varkappa_{Z} F_{2}^{\gamma Z}+\left[\left(g_{V}^{e} g_{V}^{e}+g_{A}^{e} g_{A}^{e}\right) \pm 2 P_{e} g_{V}^{e} g_{A}^{e}\right] \varkappa_{Z}^{2} F_{2}^{Z}, \\
& \tilde{F}_{3}^{ \pm}=-\left(g_{A}^{e} \pm P_{e} g_{V}^{e}\right) \varkappa_{Z} F_{3}^{\gamma Z}+\left[2 g_{V}^{e} g_{A}^{e} \pm P_{e}\left(g_{V}^{e} g_{V}^{e}+g_{A}^{e} g_{A}^{e}\right)\right] \varkappa_{Z}^{2} F_{3}^{Z},
\end{aligned}
$$

where $P_{e}$ is the longitudinal polarization of the electron beam. A similar decomposition exists for $\tilde{F}_{L}$. The coefficient $\varkappa_{Z}$ accounts for the $Z$-boson propagator and the normalization 
of the weak relative to the electromagnetic interaction. In the on-shell renormalization scheme it is calculated from the weak boson masses $m_{Z}$ and $m_{W}$ at LO as

$$
\varkappa_{Z}\left(Q^{2}\right)=\frac{Q^{2}}{Q^{2}+m_{Z}^{2}} \frac{1}{4 \sin ^{2} \theta_{W} \cos ^{2} \theta_{W}},
$$

using $\sin ^{2} \theta_{W}=1-\cos ^{2} \theta_{W}=1-m_{W}^{2} / m_{Z}^{2}$. Higher-order corrections are included through an additional factor proportional to $1 /(1-\Delta r)$ [6]. In the on-shell scheme also the Fermi constant $G_{\mathrm{F}}$ is a prediction. Electroweak couplings at the hadronic vertex are included in the structure functions and one can write them, in the naive quark-parton model, in terms of quark and anti-quark parton distribution functions, $q(x)$ and $\bar{q}(x)$,

$$
\begin{aligned}
{\left[F_{2}, F_{2}^{\gamma Z}, F_{2}^{Z}\right] } & =x \sum_{q}\left[Q_{q}^{2}, 2 Q_{q} g_{V}^{q}, g_{V}^{q} g_{V}^{q}+g_{A}^{q} g_{A}^{q}\right]\{q+\bar{q}\} \\
x\left[F_{3}^{\gamma Z}, F_{3}^{Z}\right] & =x \sum_{q}\left[2 Q_{q} g_{A}^{q}, 2 g_{V}^{q} g_{A}^{q}\right]\{q-\bar{q}\}
\end{aligned}
$$

where $Q_{f}$ denotes the electric charge of a fermion $f$ (with $f=e, q$ and $q=u, d$ ). The vector and axial-vector couplings of a fermion to the $Z$-boson, $g_{V}^{f}$ and $g_{A}^{f}$, are given by

$$
\begin{aligned}
& g_{A}^{f}=\sqrt{\rho_{\mathrm{NC}, f}} I_{\mathrm{L}, f}^{3}, \\
& g_{V}^{f}=\sqrt{\rho_{\mathrm{NC}, f}}\left(I_{\mathrm{L}, f}^{3}-2 Q_{f} \kappa_{f} \sin ^{2} \theta_{W}\right),
\end{aligned}
$$

where $I_{\mathrm{L}, f}^{3}$ is the third component of the weak-isospin of the fermion $f$. The form factors $\rho_{\mathrm{NC}, f}$ and $\kappa_{f}$ are unequal to unity beyond the LO and include higher-order corrections due to the $\gamma Z$ mixing and $Z$ self-energy corrections [7-9]. They are scale dependent and also include flavor dependent contributions beyond the universal correction factors.

Subject of interest in EW physics are the Born-level quantities, as well as potential modifications to the higher-order virtual corrections, which could originate from new physics.

\section{Methodology}

For our purpose of studying the sensitivity of future inclusive NC and CC DIS measurements to EW parameters, we simulate double-differential cross section measurements including a full set of statistical and systematic uncertainties. The procedure employs a numerical simulation procedure [10] and follows closely the one for the $\mathrm{LHeC}[2,4]$. A full suite of all cross section measurements is important, since also the PDFs are determined simultaneously from these data. The simulated data are summarized in Tab. 1.

$\mathrm{NC}$ and CC DIS cross section measurements are simulated in $e^{+} p$ and $e^{-} p$ operation mode and different lepton-beam polarization states for the nominal FCC-hh proton-beam 
energy of $E_{p}=50 \mathrm{TeV}$. A data set with reduced integrated luminosity is simulated for $E_{p}=7 \mathrm{TeV}$, which will be used for $F_{L}$ measurements. The kinematic range is assumed to be $Q^{2} \geq 3.5 \mathrm{GeV}^{2}$ in $\mathrm{NC}$, and $Q^{2} \geq 50 \mathrm{GeV}^{2}$ in $\mathrm{CC}$. Altogether 1117 cross section values are simulated for NC and CC DIS. Due to computational reasons, we have merged always $2 \times 2$ data points, so that these are representative for altogether 4468 cross section measurements.

\begin{tabular}{lccc}
\hline$E_{p}[\mathrm{TeV}]$ & $P_{e}$ & Charge & $\mathcal{L}\left[\mathrm{ab}^{-1}\right]$ \\
\hline 50 & -0.8 & -1 & 2 \\
50 & +0.8 & -1 & 0.5 \\
50 & 0 & +1 & 0.2 \\
7 & -0.8 & -1 & 0.2 \\
\hline
\end{tabular}

Table 1: Simulated data sets for different proton beam energies $E_{p}$, electron beam polarization $P_{e}$, electron or positron beam, and prospected integrated luminosity $\mathcal{L}$. All data will become available for lepton beam energies of $E_{e}=60 \mathrm{GeV}$ and for NC and CC DIS.

\begin{tabular}{lc}
\hline Source of uncertainty & Uncertainty \\
\hline Scattered electron energy scale $\Delta E_{e}^{\prime} / E_{e}^{\prime}$ & $0.1 \%$ \\
Scattered electron polar angle & $0.1 \mathrm{mrad}$ \\
Hadronic energy scale $\Delta E_{h} / E_{h}$ & $0.5 \%$ \\
Radiative corrections & $0.3 \%$ \\
Photoproduction background (for $y>0.5)$ & $1.0 \%$ \\
Global efficiency error & $0.5 \%$ \\
Luminosity uncertainty & $1.0 \%$ \\
Statistical uncertainty & $\geq 0.1 \%$ \\
\hline
\end{tabular}

Table 2: Assumptions used in the simulation of the DIS cross sections for the size of uncertainties from various sources. The top three are calibration uncertainties which are transported to provide correlated systematic cross section errors. The lower five values are uncertainties of the cross section caused by various sources.

The data include a set of the most important experimental uncertainties. These are summarised in Tab. 2. The statisical uncertainties are set to a minimum value of $0.1 \%$. The hadronic energy scale uncertainty of $0.5 \%$ becomes important for CC and for NC DIS at low- $y$, where the electron reconstruction method worsens. Heavy-flavor cross sections are not included in our study, although they may yield an interesting sensitivity to anomalous $Z b \bar{b}$ couplings [11].

The expected uncertainties in the electroweak parameter of interest are determined in a combined fit together with the PDFs. The PDFs are parameterised with five functions at a scale of $Q_{0}=1.38 \mathrm{GeV}$, and after the application of sum rules 13 free parameters are associated to the PDFs and determined from the FCC-eh pseudo-data. The structure functions are calculated in NNLO QCD using the program QCDNUM [12], the electroweak 1-loop corrections are implemented in the program EPRC [13], and the cross section calculation is implemented in a private code. The program Minuit [14] is used for the calculation of the Hesse matrix of the Azimov fit, and further technical details are given in Ref. [4]. This kind of algorithm is termed PDF $+E W$ fit in the following, where $E W$ will be replaced with the parameter(s) of interest. As a consequence of the simultaneous determination of the PDFs and the EW parameters, the prospected uncertainties will always 
include the experimental and PDF-related uncertainties. The latter, however, may become comparatively small, since the PDFs are predominantly constrained from low- $Q^{2}$ data. In contrast, high- $Q^{2}$ data are particularly sensitive to EW parameters.

The results from FCC-eh pseudo data will be compared with expectations from $\mathrm{LHeC}$ [4]. These are available for two electron-beam energy configurations of 50 or $60 \mathrm{GeV}$ and will be termed $\mathrm{LHeC}-50$ and $\mathrm{LHeC}-60$, respectively.

\section{Precision electroweak physics with inclusive NC and CC DIS}

In the following results from the PDF+EW fits to the simulated FCC-eh pseudo-data are presented. The expected uncertainties include the experimental uncertainties and PDFrelated uncertainties.

\subsection{Weak-neutral-current couplings of the first generation quarks}

The couplings of fermions to weak-neutral-currents can generically be considered as free parameters. While the couplings of the leptons and heavy quarks were measured at LEP/SLD with high precision [15], the couplings of the first generation quarks have sizeable experimental uncertainties still today. In the Standard Model, these couplings are determined as vector and axial-vector couplings (c.f. eqs. (7) and (8)). A meaningful test of the Standard Model is performed by determining $g_{V}$ and $g_{A}$ and comparing them with the SM prediction. High experimental precision is desirable, since in the SM higher-order corrections modify the vector and axial-vector couplings, and new physics may alter these virtual corrections.

We perform a PDF $+g_{A}^{d}+g_{V}^{d}+g_{A}^{u}+g_{V}^{u}$ fit to study the determination the NC couplings of the $u$-type and $d$-type quarks. The contributions from strange quarks are smaller than $10 \%$ and those from heavier quarks are negligible. Thus the parameters obtained in this fit can be interpreted as the NC couplings of the first generation quarks. The lepton couplings are kept fixed at their predicted values since they were tested with high precision at LEP/SLD. The expected uncertainties from a fit to $\mathrm{LHeC}$ or FCC-eh pseudo-data are presented in Figure 1. The uncertainties are found to be smaller by orders of magnitudes than today's best measurements [15-17]. This is due to the high luminosity and the high center-of-mass energy of the LHeC and FCC-eh, such that weak interactions of the valence quarks are probed.

\subsection{The effective weak mixing angle}

The weak mixing angle is the central parameter in the electroweak theory [18]. It determines the value of $g_{V}$, but also higher-order corrections become relevant therein. As 

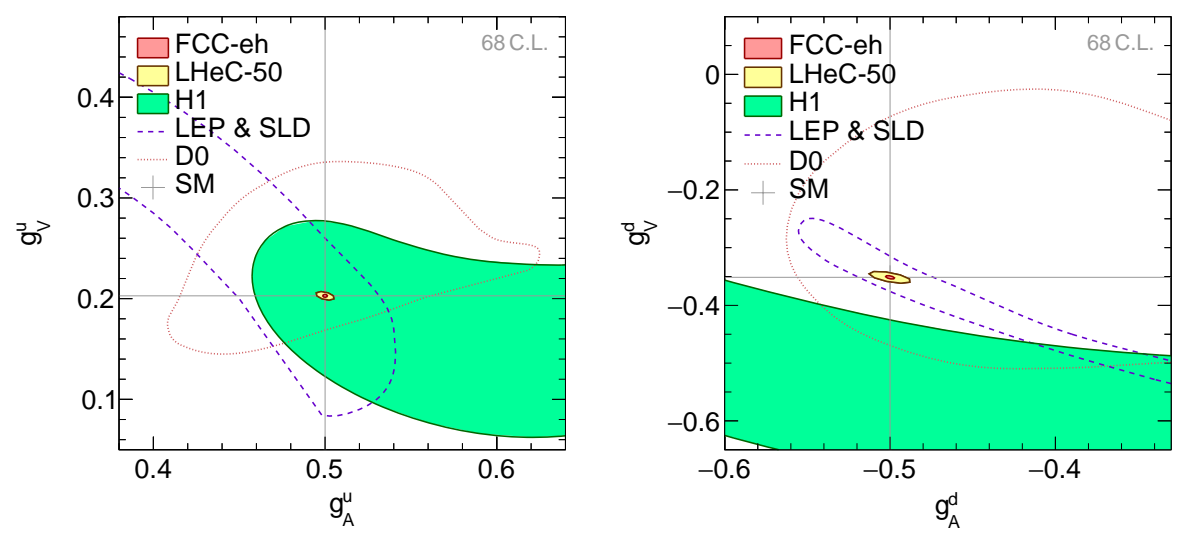

Figure 1: Prospected uncertainties of the weak-neutral-current vector and axial-vector couplings of first generation quarks (up-type, and down-type) to the Z-boson at $68 \%$ confidence level. Left: down-type quarks. Right: up-type quarks. The prospected uncertainties are compared with results from LEP+SLD [15], D0 [16] and H1 [17]. The SM expectations are at the crossing of the horizontal and vertical lines.

a consequence, usually the effective weak mixing angle is studied, which combines the Born-level expression and higher-order corrections as

$$
\sin ^{2} \theta_{W, f}^{\mathrm{eff}}\left(\mu^{2}\right)=\kappa_{f}\left(\mu^{2}\right) \sin ^{2} \theta_{W} .
$$

Its value at $\mu^{2}=m_{Z}^{2}$ is subject to measurements at LEP/SLD, Tevatron and the LHC. We estimate the uncertainty of $\sin ^{2} \theta_{\mathrm{W}, \ell}^{\text {eff }}$ in a $\mathrm{PDF}+\sin ^{2} \theta_{\mathrm{W}, \ell}^{\mathrm{eff}}$ fit. In order to map the values of $\sin ^{2} \theta_{\mathrm{W}, f}^{\mathrm{eff}}$ that contribute in the spacelike calculation of the NC DIS cross sections $\left(\mu^{2}=-Q^{2}\right)$ to the definition of $\sin ^{2} \theta_{\mathrm{W}, \ell}^{\text {eff }}$ from LEP/SLD, we utilize scale- and flavordependent correction factors and assume the validity of the SM for that purpose. We find, that from FCC-eh pseudo-data, the value of $\sin ^{2} \theta_{\mathrm{W}, \ell}^{\mathrm{eff}}$ can be determined with an uncertainty of

$$
\Delta \sin ^{2} \theta_{\mathrm{W}, \ell}^{\mathrm{eff}}= \pm 0.00011 .
$$

This uncertainty includes experimental and PDF-related uncertainties, and the results are compared with presently available measurements and the PDG-prediction [18] in Fig. 3 (right).

A determination of $\sin ^{2} \theta_{\mathrm{W}, \ell}^{\text {eff }}$ is further studied for LHeC data, and in a combined analysis of LHeC and FCC-eh data. Results are presented in Tab. 3. It is observed, that the uncertainties will be competitive with the combination of the LEP+SLD data, but determined here from a single experiment and a single process only. At this level of precision, theoretical uncertainties may be of non-negligible size, and should be assessed once real data are available.

It should be stressed that the determination of $\sin ^{2} \theta_{\mathrm{W}, \ell}^{\mathrm{eff}}$ represents a precision test of the $\mathrm{SM}$ at the quantum level and many BSM models predict modifications not only of $\sin ^{2} \theta_{\mathrm{W}, \ell}^{\mathrm{eff}}$ 


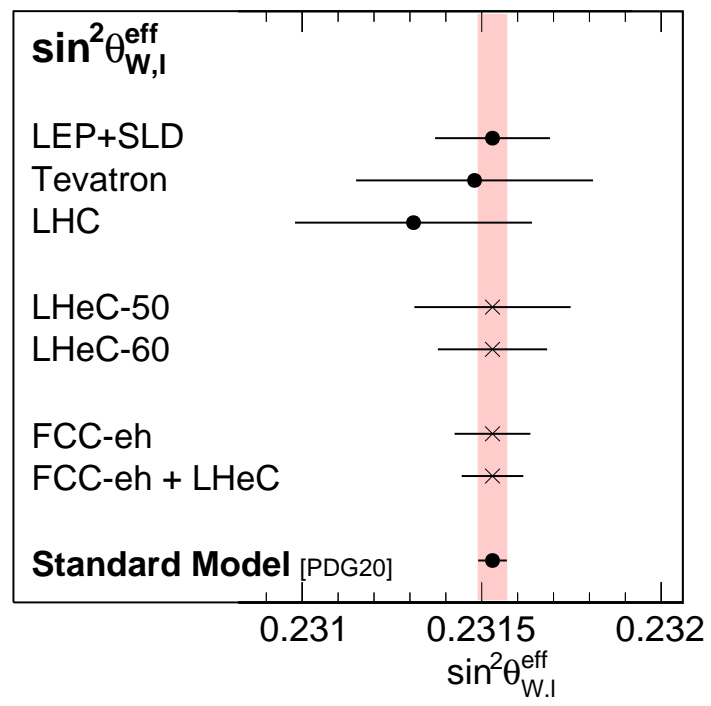

Figure 2: Prospected uncertainties for the determination of $\sin ^{2} \theta_{\mathrm{W}, \ell}^{\mathrm{eff}}$ from LHeC or FCC-eh in comparison with present measurements and the SM expectation.

\begin{tabular}{lcc}
\hline Facility & $\Delta \sin ^{2} \theta_{\mathrm{W}, \ell}^{\mathrm{eff}}$ & $\Delta m_{W}$ \\
\hline LHeC-50 & \pm 0.00021 & $\pm 8 \mathrm{MeV}$ \\
LHeC-60 & \pm 0.00015 & $\pm 6 \mathrm{MeV}$ \\
FCC-eh & \pm 0.00011 & $\pm 4.5 \mathrm{MeV}$ \\
FCC+LHeC & \pm 0.000086 & $\pm 3.6 \mathrm{MeV}$ \\
\hline
\end{tabular}

Table 3: Comparision of the prospected uncertainties on the determination of the effective weak mixing angle $\sin ^{2} \theta_{\mathrm{W}, \ell}^{\text {eff }}$ for different collections of future DIS data. The right column shows the prospected uncertainties in $m_{W}$ when employing the on-shell renormalization scheme.

but also of the form factors $\rho_{\mathrm{NC}, f}$. Unfortunately, in DIS, the contributions from the latter cannot be removed by using asymmetries, like the forward-backward asymmetry, as it can be done for $Z$-pole measurements. Therefore, a simultaneous determination of the form factors $\rho_{\mathrm{NC}, f}$ and $\sin ^{2} \theta_{\mathrm{W}, \ell}^{\text {eff }}$ is of interest and it is studied in terms of a $\mathrm{PDF}+\rho_{\mathrm{NC}, f}^{\prime}+\kappa_{f}^{\prime}$ fit, where the primed parameters represent multiplicative anomalous form factors describing physics beyond the SM, i.e. they are unity in the SM [17]. The prospected uncertainties from the FCC-eh are compared with those from the $\mathrm{LHeC}$ and with what was obtained from the combination of LEP and SLD data [15]. It is observed, that the level of precision which can be achieved in DIS is comparable with that from $e^{+} e^{-}$experiments. 

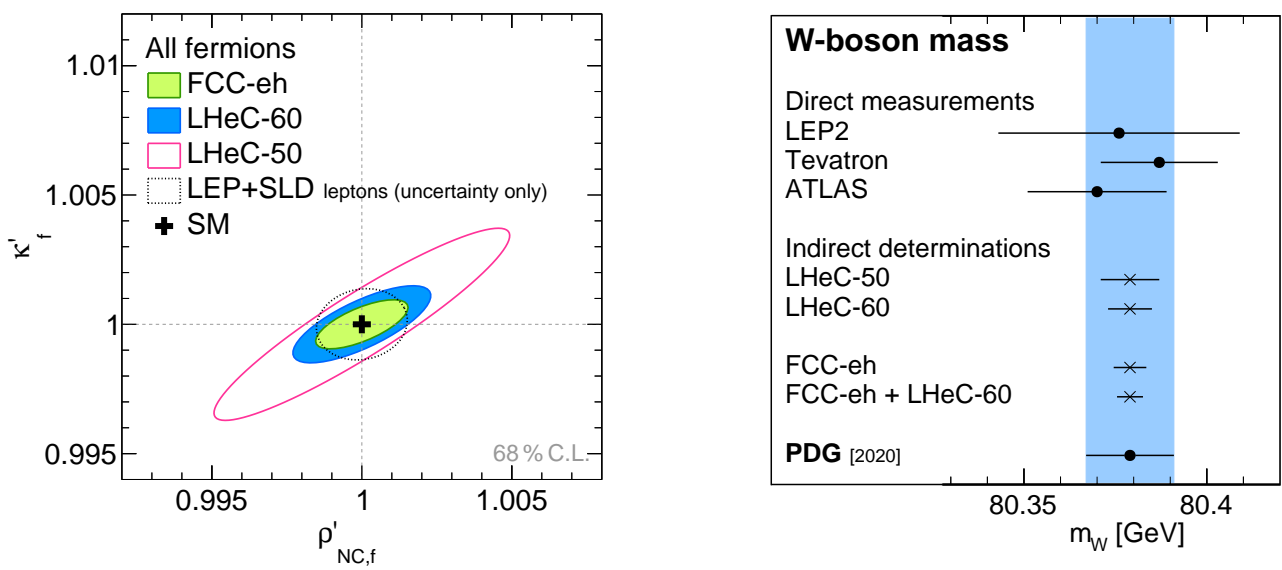

Figure 3: Left: Prospected uncertainties for the determination of $\rho_{\mathrm{NC}, f}$ and $\sin ^{2} \theta_{\mathrm{W}, \ell}^{\mathrm{eff}}$, expressed in terms of anomalous form factors $\rho_{\mathrm{NC}, f}^{\prime}$ and $\kappa_{f}^{\prime}$, in comparison with achieved uncertainties from the LEP/SLD combination at a confidence level of $68 \%$. Right: Prospected uncertainties for $m_{W}$ when determined in the on-shell scheme in comparison with direct measurements.

\subsection{The $W$-boson mass in the on-shell scheme}

In the on-shell renormalization scheme the $W$-boson mass is a Born-level parameter together with $\alpha$ and $m_{Z}$, and consequently any derived quantity, like e.g. $G_{\mathrm{F}}$ or $\sin ^{2} \theta_{W}$, depend on the value of $m_{W}$. A PDF $+m_{W}$ fit in the on-shell scheme is studied, which represents a consistency test of electroweak theory at hand of a single parameter only, when compared to direct measurements. The prospected uncertainties in $m_{W}$ are summarized in Tab. 3 and displayed in Fig. 3. We find, that at the FCC-eh, the value of $m_{W}$ can be determined from the inclusive DIS data with an uncertainty of

$$
\Delta m_{W}= \pm 4.5 \mathrm{MeV}
$$

The expectations for the LHeC are discussed in Refs. [2, 4]. From a simultaneous analysis of $\mathrm{LHeC}$ and FCC-eh data, the prospected uncertainty is $\Delta m_{W}= \pm 3.6 \mathrm{MeV}$. It is observed that the measurements at the $\mathrm{LHeC}$ or FCC-eh yield smaller uncertainties than any direct measurement today, as well as the indirect constraints from the global electroweak fit [1921]. However, additional theoretical uncertaities will have to be taken into account at that level of precision, and, for instance, the uncertainty due to the uncertainty of the top-quark mass, $\Delta m_{t}= \pm 0.5 \mathrm{GeV}$, will contribute with an uncertainty of about $\Delta m_{W}=2.5 \mathrm{MeV}$ for the $\mathrm{LHeC}$ analysis. Also the interpretation of the value of $m_{W}$ is non-trivial. In a dedicated study it was found that the dominant contributions arise from the weak mixing angle in the NC vector couplings, whereas the sensitivity from the $W$-boson propagator in the CC DIS cross sections is comparatively small. 


\subsection{The oblique parameters $S, T$ and $U$}

Additional contributions from modifications of the SM can generically be considered in terms of the oblique parameters $S, T$ and $U$ [22], which represent non-SM contributions to the gauge boson self energies, and are particularly suitable to describe the effects due to new heavy particles. We perform fits of two of these parameters together with parameters of the PDFs. The prospected uncertainties of two oblique parameters are displayed in Fig. 4 at a confidence level of $68 \%$ for LHeC and FCC-eh simulated data. The oblique parameters can be determined with uncertainties of about \pm 0.1 . The combined analysis of $\mathrm{NC}$ and CC DIS data would also allow to determine all three oblique parameters at a time, albeit with large uncertainties due to correlations.
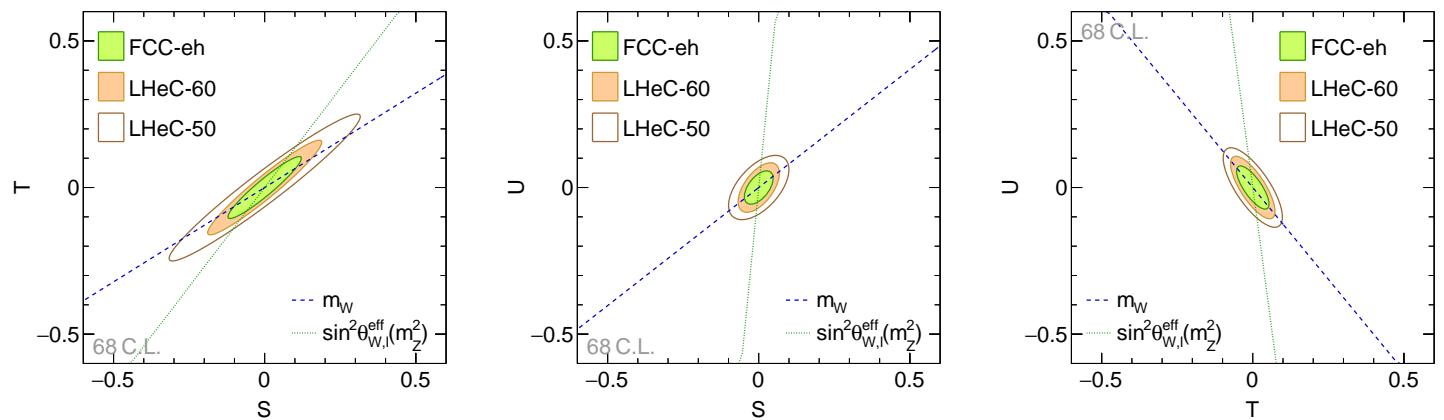

Figure 4: Prospected uncertainties on the oblique parameters $S, T$ and $U$ when two parameters are determined at a time (together with the PDFs) from LHeC or FCC-eh pseudo-data. The parameters are defined in the modified on-shell scheme and the lines indicate the SM values of $m_{W}$ and $\sin ^{2} \theta_{\mathrm{W}, \ell}^{\mathrm{eff}}$.

These studies were done in the modified on-shell scheme [23], where $G_{\mathrm{F}}$ is a Born-level quantity and $m_{W}$ a prediction. The on-shell renormalization scheme results in stronger correlations of the oblique parameters.

\subsection{The running of the weak mixing angle}

The scale dependence of the weak mixing angle is given through the renormalization scheme and loop corrections, and it is closely related to the underlying gauge structure of electroweak theory. Many BSM models will yield a change of the scale dependence of $\sin ^{2} \theta_{\mathrm{W}, f}^{\mathrm{eff}}$ through modifications to the virtual corrections. However, precision measurements are available only at the $Z$-pole, or with moderate precision at much lower scales, which is why the running of $\sin ^{2} \theta_{\mathrm{W}, f}^{\mathrm{eff}}$ is largely not tested by experiments until today.

The spacelike momentum transfer in DIS provides a unique opportunity to perform a study of the scale-dependence of the weak mixing angle. We perform a sensitivity study, by performing a single fit to inclusive NC and CC DIS data and determining $\sin ^{2} \theta_{\mathrm{W}, f}^{\mathrm{eff}}$ at 
different values of $Q^{2}$, together with the parameters of the PDFs. Again, only the effective weak mixing angle in the vector-couplings are tested, while other parameters like $\rho_{\mathrm{NC}}, \rho_{C C}$ or $\Delta r$ are taken from the SM.

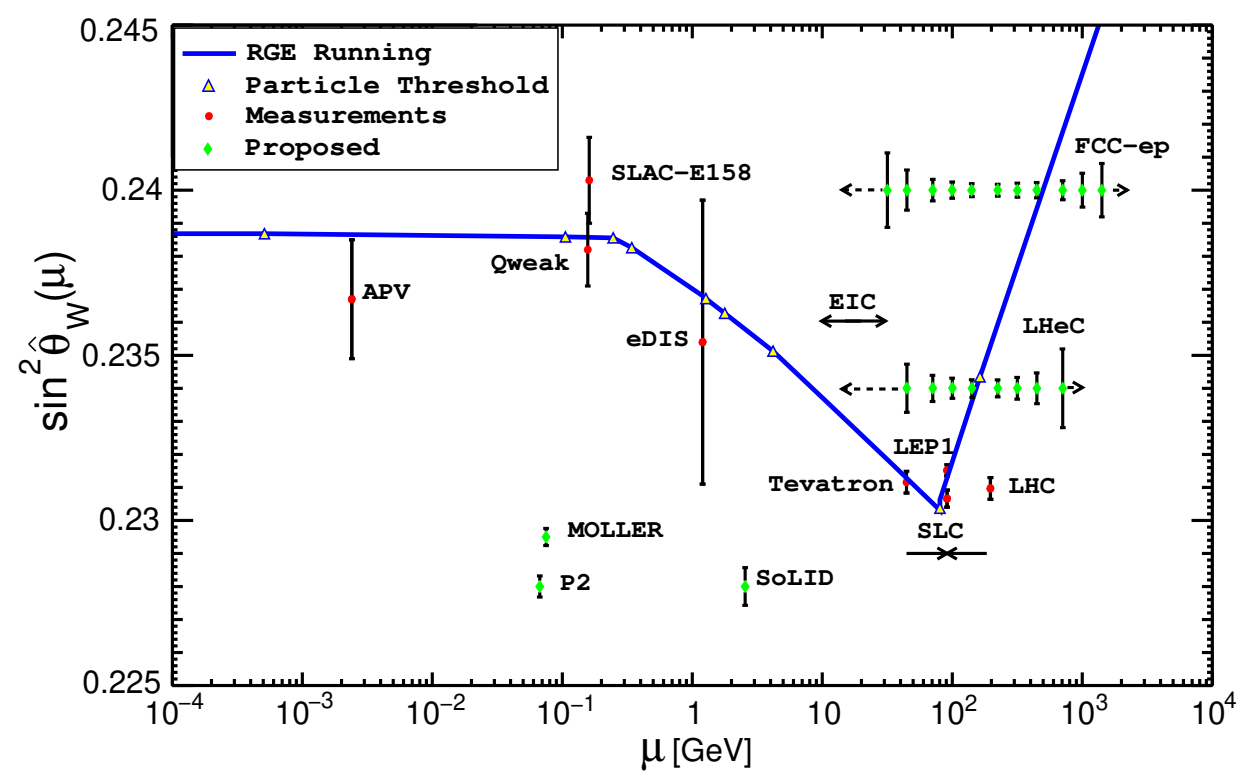

Figure 5: Present and future measurements of the running of the weak mixing angle in the $\overline{\mathrm{MS}}$ scheme as a function of $\mu$. The prospected uncertainties from the LHeC and FCC-eh are displayed. All future facilities are displayed with green markers and have an arbitrary vertical offset for better visibility, whereas the $Z$-pole measurements are horizontally displaced. (Credits/courtesy: J. Erler, R. Ferro-Hernandez and X. Zheng; updated from Ref. [24, 25]).

The expected uncertainties for $\mathrm{LHeC}$ and FCC-eh are displayed in Fig. 5 and compared to available measurements and the expectation in the $\overline{\mathrm{MS}}$ scheme. Small correction factors to translate the on-shell definition to the $\overline{\mathrm{MS}}$ definition of $\sin ^{2} \theta_{W}$ will not visibly change the uncertainty estimate. It is found, that the LHeC and FCC-eh are able to measure the running of the weak mixing angle in the range from about $30 \mathrm{GeV}$ up to the $\mathrm{TeV}$ scale with considerable precision. Experiments at the LHeC and FCC-eh would represent the first measurements, where the running is directly tested in a single experiment, and the first indication for a scale dependence at the electroweak scale and above.

\subsection{Precision electroweak physics with charged currents}

Precision electroweak physics in charged-current interactions is a poorly studied field, since measurements of charged currents have commonly only low precision due to the presence of a neutrino that escapes undetected. In contrast, in DIS, the kinematic variables $Q^{2}, x$ and $y$ can be meaured from the incoming electron beam and the hadronic final state also for charged-current DIS, which allows for precise measurements in a large kinematic region. 
Similar to the NC case, EW corrections are included in the cross section calculation through two form factors $\rho_{\mathrm{CC}, e q}$ and $\rho_{\mathrm{CC}, e \bar{q}}[17,26,27]$. We perform a study for their determinations, by introducing corresponding multiplicative anomalous form factors $\rho_{\mathrm{CC}, e q}^{\prime}$ and $\rho_{\mathrm{CC}, e \bar{q}}^{\prime}[17]$, which are unity in the SM.
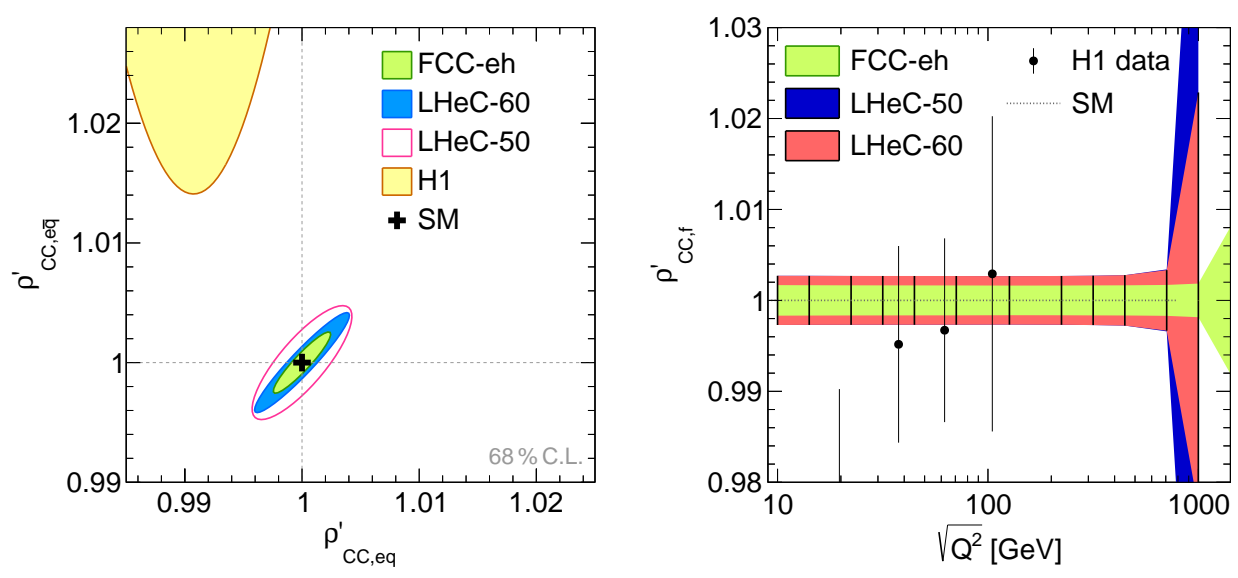

Figure 6: Left: Prospected uncertainties of CC coupling parameters $\rho_{\mathrm{CC}, e q}^{\prime}$ and $\rho_{\mathrm{CC}, e \bar{q}}^{\prime}$ from LHeC or FCC-eh data compared with the only available measurement from H1 [17]. Right: Prospected uncertainties for the scale dependent determination of $\rho_{\mathrm{CC}}^{\prime}$ in comparison to $\mathrm{H} 1$.

The prospected uncertainties of $\rho_{\mathrm{CC}, e q}^{\prime}$ and $\rho_{\mathrm{CC}, e \bar{q}}^{\prime}$ are determined in a $\mathrm{PDF}+\rho_{\mathrm{CC}, e q}^{\prime}+\rho_{\mathrm{CC}, e \bar{q}}^{\prime}$ fit and are displayed in Fig. 6 (left) at a confidence interval of $68 \%$. It is observed that virtual corrections in CC interactions can be tested at the per mille level, greatly exceeding the pioneering measurements from $\mathrm{H} 1$ [17].

The $\rho_{\mathrm{CC}}$ form factors are scale dependent, and it is expected that BSM contributions will modify them through contributions to these virtual corrections. The uncertainty estimates of $\rho_{\mathrm{CC}}$ (using $\rho_{\mathrm{CC}, e q}^{\prime}=\rho_{\mathrm{CC}, e \bar{q}}^{\prime}=\rho_{\mathrm{CC}}$ ) at different scales $Q^{2}$ is presented in Fig. 6 (right) and it is seen that with FCC-eh uncertainties at the level of a few per mille are obtained over a large range of $\sqrt{Q^{2}}$, up to the $\mathrm{TeV}$ regime. This is a very unique measurement.

\section{Conclusions and Outlook}

We have presented a study of the determination of parameters of the Electroweak Theory using pseudo-data of the proposed $\mathrm{LHeC}$ and FCC-eh electron-proton colliders. The data are inclusive neutral-current and charged-current DIS cross sections and include a full set of statistical and systematic uncertainties. The study employs NNLO QCD predictions for the structure functions and NLO EW predictions for the partonic cross sections. Parameters are determined in a combined PDF plus EW fit. 
It is found, that several quantities in the EW sector can be determined with very high precision, and values of the effective weak mixing angle or the $W$-boson mass may even exceed the precision of the combined measurements from LEP/SLD. Since the interaction in DIS is mediated in the spacelike regime, all these measurements are unique. In fact, several aspects of the EW theory can only be studied in DIS in the $t$-channel. This includes the light-quark weak-neutral-current couplings, the running of the weak mixing angle from the electroweak up to the $\mathrm{TeV}$ energy scale, and precision electroweak physics in charged-current interactions.

\section{References}

[1] LHeC Study Group, J. Abelleira Fernandez et al., "A Large Hadron Electron Collider at CERN: Report on the Physics and Design Concepts for Machine and Detector," J. Phys. G 39 (2012) 075001, arXiv: 1206.2913

[2] LHeC, FCC-he Study Group Collaboration, P. Agostini et al., "The Large Hadron-Electron Collider at the HL-LHC," arXiv: 2007. 14491.

[3] FCC Study Group, A. Abada et al., "FCC Physics Opportunities: Future Circular Collider Conceptual Design Report Volume 1," Eur. Phys. J. C 79 (2019) 474.

[4] D. Britzger, M. Klein and H. Spiesberger, "Electroweak physics in inclusive deep inelastic scattering at the LHeC," Eur. Phys. J. C 80 (2020) 831, arXiv: 2007.11799.

[5] M. Klein and T. Riemann, "Electroweak interactions probing the nucleon structure," Z. Phys. C24 (1984) 151.

[6] A. Sirlin, "Radiative corrections in the $\mathrm{SU}(2)_{\mathrm{L}} \times \mathrm{U}(1)$ theory: A simple renormalization framework," Phys. Rev. D22 (1980) 971.

[7] M. Böhm and H. Spiesberger, "Radiative corrections to neutral current deep inelastic lepton nucleon scattering at HERA energies," Nucl. Phys. B294 (1987) 1081.

[8] D. Yu. Bardin, C. Burdik, P. C. Khristova and T. Riemann, "Electroweak radiative corrections to deep inelastic scattering at HERA, neutral current scattering," Z. Phys. C42 (1989) 679.

[9] W. Hollik, D. Yu. Bardin, J. Blümlein, B. A. Kniehl, T. Riemann and H. Spiesberger, "Electroweak parameters at HERA: Theoretical aspects," in Workshop on physics at HERA Hamburg, Germany, October 29-30, 1991, 1992.

[10] J. Blümlein and M. Klein, "Kinematics and resolution at future e p colliders," in 1990 DPF Summer Study on High-energy Physics: Research Directions for the Decade (Snowmass 90), 91990.

[11] B. Yan, Z. Yu and C. P. Yuan, "The anomalous Zbb- couplings at the HERA and EIC," Phys. Lett. B 822 (2021) 136697, arXiv: 2107.02134.

[12] M. Botje, "QCDNUM: fast QCD evolution and convolution," Comput. Phys. Commun. 182 (2011) 490, arXiv: 1005 . 1481. [Erratum: arXiv: 1602 .08383].

[13] H. Spiesberger, "EPRC: A program package for electroweak physics at HERA," in Future physics at HERA. Proceedings, Workshop, Hamburg, Germany, September 25, 1995-May 31, 1996. Vol. 1, 2, 1995.

[14] F. James and M. Roos, "Minuit: A system for function minimization and analysis of the parameter errors and correlations," Comput. Phys. Commun. 10 (1975) 343.

[15] DELPHI, ALEPH, SLD, OPAL and L3 Collaborations and SLD and LEP Electroweak Working 
Groups, S. Schael et al., "Precision electroweak measurements on the $Z$ resonance," Phys. Rept. 427 (2006) 257, arXiv : hep-ex/0509008.

[16] D0 Collaboration, V. M. Abazov et al., "Measurement of $\sin ^{2} \theta_{\mathrm{eff}}^{\ell}$ and $Z$-light quark couplings using the forward-backward charge asymmetry in $p \bar{p} \rightarrow Z / \gamma^{*} \rightarrow e^{+} e^{-}$events with $\mathcal{L}=5.0 \mathrm{fb}^{-1}$ at $\sqrt{s}=1.96$ TeV," Phys. Rev. D84 (2011) 012007, arXiv: 1104.4590.

[17] H1 Collaboration, V. Andreev et al., "Determination of electroweak parameters in polarised deep-inelastic scattering at HERA," Eur. Phys. J. C78 (2018) 777, arXiv: 1806. 01176.

[18] J. Erler and A. Freitas, "Electroweak Model and Constraints on New Physics," in Review of Particle Physics, PTEP 2020 (2020) 8, 083C01, P. A. Zyla et al. (eds.), 2020.

[19] J. Haller, A. Hoecker, R. Kogler, K. Mönig, T. Peiffer and J. Stelzer, "Update of the global electroweak fit and constraints on two-Higgs-doublet models," Eur. Phys. J. C78 (2018) 675, arXiv: 1803.01853.

[20] J. de Blas, M. Ciuchini, E. Franco, S. Mishima, M. Pierini, L. Reina and L. Silvestrini, "Electroweak precision observables and Higgs-boson signal strengths in the Standard Model and beyond: present and future," JHEP 12 (2016) 135, arXiv: 1608.01509.

[21] J. Erler and M. Schott, "Electroweak Precision Tests of the Standard Model after the Discovery of the Higgs Boson,” Prog. Part. Nucl. Phys. 106 (2019) 68, arXiv: 1902.05142.

[22] M. E. Peskin and T. Takeuchi, "Estimation of oblique electroweak corrections," Phys. Rev. D46 (1992) 381.

[23] W. J. Marciano and A. Sirlin, "Radiative corrections to neutrino induced neutral current phenomena in the SU(2) $\times$ U U(1) theory," Phys. Rev. D22 (1980) 2695. [Erratum: Phys. Rev. D31 (1985) 213].

[24] J. Erler and M. J. Ramsey-Musolf, "The Weak mixing angle at low energies," Phys. Rev. D 72 (2005) 073003, arXiv: hep-ph/0409169.

[25] J. Erler and R. Ferro-Hernández, "Weak Mixing Angle in the Thomson Limit," JHEP 03 (2018) 196, arXiv: 1712.09146.

[26] M. Böhm and H. Spiesberger, "Radiative corrections to charged current deep inelastic electron proton scattering at HERA," Nucl. Phys. B304 (1988) 749.

[27] D. Yu. Bardin, K. C. Burdik, P. K. Khristova and T. Riemann, "Electroweak radiative corrections to deep inelastic scattering at HERA, charged current scattering," Z. Phys. C44 (1989) 149. 